\title{
Aptitude de trois souches de moisissures à produire des enzymes extracellulaires en milieu solide au Burkina Faso
}

\author{
Hamidou COMPAORÉ ${ }^{* 1.2}$, Hagrétou SAWADOGO-LINGANI2, Serge SAMANDOULOUGOU², Filbert \\ GUIRA ${ }^{1}$, Aly SAVADOGO ${ }^{1}$ and Alfred S. TRAORE ${ }^{1}$ \\ ${ }^{1}$ Research Center for Biological Alimentary and Nutritional Sciences. Research and Training Unit. Life and Earth \\ Sciences. University of Ouaga I Pr Joseph Ki-Zerbo. 03 BP 7131 Ouagadougou 03. Ouagadougou. Burkina Faso. \\ 2Département Technologie Alimentaire (DTA/IRSAT/CNRST). 03 BP 7047 Ouagadougou 03. Ouagadougou. Burkina \\ Faso. \\ * Corresponding author : Hamidou COMPAORE. Email : hamidoucom@yahoo.fr; Tel : (+226) 76360809 ; Fax : \\ (+226) 25368728
}

Original submitted in on $1^{\text {st }}$ November 2016. Published online at www.m.elewa.orgon $28^{\text {th }}$ February 2017 http://dx.doi.org/10.4314/jab.v110i1.7

\section{RÉSUMÉ}

Objectif : La présente étude a pour objectif la caractérisation physiologique de trois souches de moisissures $\mathrm{S}_{1}$, $\mathrm{S}_{2}$ et $\mathrm{S}_{3}$ isolées respectivement du maïs, de l'arachide et du riz au biais de leur capacité à produire des enzymes extracellulaires.

Méthodologie et résultats : Au total, soixante-seize (76) échantillons d'aliments locaux ont été collectés dans des marchés centraux de trois villes du Burkina Faso. Deux cent soixante-douze (272) souches de moisissures ont été isolées et soumises au criblage indirect de production d'enzymes extracellulaires. II consiste à estimer la production par mesure de la vitesse de croissance apicale des souches fongiques sur des milieux solides supplémentés par différents substrats carbonés. Le criblage a révélé l'activité protéolitique, amylolitiques, tannasiques, lipolitique et cellulosique de trois souches de moisissures. Selon les clés d'identification de Raper \& Fennell (1965) et Pitt (1985), ces trois souches $S_{1}, S_{2}$ et $S_{3}$ sont respectivement Aspergillus fumigatus, Aspergillus flavus et Penicillium citrinum.

Conclusion et application : Les micro-organismes en général et les champignons en particulier constituent des sources d'enzymes plus facilement exploitables que celles des végétaux ou les animaux à cause de la simplicité de purification. Les enzymes fongiques restent toujours les outils clés de la biotechnologie et reflètent de plus en plus l'importance et le rôle infini des moisissures dans les différentes applications alimentaires. Parmi ces enzymes, les lipases, les phytases et les tannases sont perçues comme étant des enzymes importantes en industrie. Cependant, le principal obstacle qui freine les applications potentielles de ces enzymes est leur coût. Des souches locales productrices d'intéressantes enzymes pourraient contribuer à l'amoindrir.

Mots clés : enzymes extracellulaires, aliments locaux, moisissures, milieu solide, croissance apicale 
The ability of three strains of fungi to produce extracellular enzymes on solid state in Burkina Faso Abstract

Objective: The aim of this study is the physiological characterization of three fungi strains $S_{1}, S_{2}$ and $S_{3}$ respectively isolated from maize, groundnuts and rice through their ability to produce extracellular enzymes. Methods and Results: A total of seventy-six (76) samples of local foods were collected in centrals markets of three cities in Burkina Faso. Two hundred and seventy-two (272) fungi strains were isolated and submitted to extracellular enzymes indirect screening production. It was consisted by measuring the apical growth rate of the fungi strains on solid media supplemented with various carbon substrates. The screening revealed the proteolytic, amylolitic, tannasic, lipolytic and cellulosic activities of three fungi strains. According to the Raper \& Fennell (1965) and Pitt (1985) identification key, these three strains $S_{1}, S_{2}$ and $S_{3}$ are respectively Aspergillus fumigatus, Aspergillus flavus and Penicillium citrinum.

Conclusion and Application: Micro-organisms in general and filamentous fungi in particular are rich sources of enzymes more easily exploitable than those of plants or animals due to the simplicity in purification. Fungal enzymes remain biotechnology key tools and reflect increasingly important and the infinity role of mold in various food applications. Among these enzymes, lipases, phytases and tannases are perceived as important enzymes in industry. However, the main obstacle hindering the potential applications of these enzymes is their cost. Local fungi strains with interesting enzymes property could help cost decreasing.

Keywords: extracellular enzymes, local foods, fungi, solid state, apical growth

\section{INTRODUCTION}

Les moisissures sont des champignons filamenteux microscopiques, ayant une application étendue en biotechnologie par leur capacité à conquérir des substrats naturels, grâce à un arsenal enzymatique très développé. Les champignons sont d'une grande importance, pas seulement en ce qui concerne la santé et l'industrie mais aussi du point de vue économique, grâce à leurs propriétés métaboliques. Ainsi, la capacité à produire des acides organiques, tels que l'acide citrique et itaconique, quelques enzymes, pigments et antibiotiques par les champignons a été exploitée (Awad, 2005; Kalyani et al., 2016). Elles élaborent une panoplie d'enzymes qui leur permettent d'utiliser plus efficacement que les bactéries les substrats les plus complexes (Sarah, 2011). Pratiquement tous les composés organiques peuvent être utilisés comme source de carbone et d'énergie par les moisissures. La plupart d'entre elles peuvent métaboliser le glucose et le saccharose avec quelques polysaccharides comme l'amidon et la cellulose (Compaoré et al., 2016a). Leur digestion doit commencer dans le milieu extérieur par des enzymes excrétées (extra cellulaires) ou liées à la paroi (Davet, 1996). Les enzymes fongiques restent toujours les outils clés de la biotechnologie et reflètent de plus en plus l'importance et le rôle infini des moisissures dans les différentes applications alimentaires. Aspergillus niger produit d'intéressantes enzymes comme les protéases, des lipases et des estérases utilisées dans différentes applications alimentaires. Les protéases sont utilisées en boulangerie tandis que les lipases servent dans la technologie des huiles. Cette souche élabore aussi la cellulase, l'amylase, l'invertase et la pectinase, utilisées principalement comme des catalyseurs biologiques en glucoserie, brasserie et pour la fabrication des boissons (Kosikowski, 1988). Selon Botton et al. (1999), environ $40 \%$ des enzymes industrielles sont d'origine fongique. Cependant, les enzymes thermostables par leurs structures et leurs fonctions sont de plus en plus sollicitées par les industries chimiques et alimentaires. Les souches thermophiles ou thermotolérantes comme Aspergillus fumigatus capable de produire des amylases et des protéases qui sont plus thermostables que les enzymes des champignons mésophiles pourrait en être la solution (Lamrani, 2009). L'objectif de cette étude est la caractérisation physiologique de trois souches de moisissures grâce à leur aptitude à se développer sur certains substrats carbonés en milieu solide. Cette croissance permet ainsi d'apprécier indirectement l'élaboration d'enzymes extra cellulaires. Les tests d'aptitude de production 
d'enzymes par fermentation en milieu solide ont

\section{MATERIEL ET METHODES}

Identification morphologique des souches isolées : L'identification morphologique des souches de moisissures s'est basée sur les caractères culturaux et la morphologie des fructifications et des spores ; les clés d'identification utilisées : Raper \& Fennell (1965) pour l'identification du genre Aspergillus, celle de Pitt (1985) pour l'identification du genre Penicillium et celle de Cooney \& Emerson (1964) pour l'identification des Champignons Filamenteux Thermophiles.

Caractérisation macroscopique: Les souches de moisissures ont été isolées et purifiées sur la gélose de Pomme de Terre Dextrose (PDA). Les caractéristiques macroscopiques suivantes ont été déterminées avec une culture de 5 jours incubées à $25^{\circ} \mathrm{C}$ dans ce milieu: forme, taille, couleur, aspect, contour et vitesse de croissance des colonies fongiques.

Caractérisation microscopique : La partie superficielle du mycélium fongique est prélevée à l'aide d'une anse, puis placée sur une lame dans une goutte d'eau distillée ou simplement entre lame/scotch. Les caractéristiques notées sont: la couleur, l'aspect et la taille des conidiophores, le nombre des conidies, la forme de la tête (aspergillaire ou pénicillaire), la présence de métules... Vitesse de croissance apicale : Pour le calcul de la vitesse de croissance apicale, le diamètre des colonies fongiques est mesuré avec un pied à coulisse toutes les 24 heures du temps d"incubation à la température convenable pour la croissance optimale des souches $S_{1}, S_{2}$ et $S_{3}$. La vitesse de croissance optimale par apicale correspond à la vitesse d'élongation du mycélium cultivé, elle s'exprime en millimètre rapportés au temps d'incubation en heure.

$V a_{\text {moy }}=\left(D_{\text {max }} / 2\right) /$ temps

Vamoy : vitesse de croissance apicale moyenne du mycélium en mm. $\mathrm{h}^{-1}$

Dmax : Diamètre de la colonie en $\mathrm{mm}$ du dernier jour

\section{RESULTATS}

Identification morphologique des souches isolées

Caractérisation macroscopique : Les souches $\mathrm{S}_{1}$ et $\mathrm{S}_{2}$ ont présenté de larges colonies de plus de 30 millimètres de diamètre en cinq jours d'incubation à $30^{\circ} \mathrm{C}$ sur la donné des résultats encourageants.

Étude de la croissance des souches de moisissures en fonction de la température (thermophilie) : L'étude de la thermophilie des souches de moisissures a été réalisée sur le milieu rapporté par Hankin \& Anagnostakis (1975). Les trois souches $S_{1}, S_{2}$ et $S_{3}$ ont été repiquées sur ce milieu et incubées pendant une semaine (Lamrani, 2009). Le gradient de températures utilisées est : $20^{\circ} \mathrm{C}$, $30^{\circ} \mathrm{C}, 37^{\circ} \mathrm{C}, 50^{\circ} \mathrm{C}$ et $55^{\circ} \mathrm{C}$. La vitesse de croissance apicale est calculée pour chaque souche.

Aptitude des souches de moisissures à produire des enzymes extracellulaires : Elle a été appréciée par la mesure de la croissance apicale des souches de moisissures sur le milieu synthétique composé de : 0,48 $\mathrm{g} / \mathrm{lde} \mathrm{CaCl}_{2}, 0,38 \mathrm{~g} / \mathrm{l}$ de $\mathrm{MgSO}_{4}, 2,27 \mathrm{~g} / \mathrm{l}$ de $\mathrm{KH}_{2} \mathrm{PO}_{4}, 0,32$ $\mathrm{g} / \mathrm{l}$ de $\mathrm{NaCl}, 6,6 \mathrm{~g} / \mathrm{l}$ de $\left(\mathrm{NH}_{4}\right)_{2} \mathrm{SO}_{4}$ et $20 \mathrm{~g} / \mathrm{l}$ d'agar. $\mathrm{Ce}$ milieu est successivement supplémenté par différentes sources de carbone : le glucose, le saccharose, l'amidon, la cellulose, l'acide phytique, l'acide tannique, les protéines (caséine) et les lipides (tween 80). Les milieux sont stérilisés à l'autoclave à $121^{\circ} \mathrm{C}$ pendant 15 minutes et coulés en boite de Pétri sauf le milieu à base de l'acide phytique qui subit une stérilisation par filtration. Trois repiquages sont effectués pour s'assurer d'une quelconque assimilation. Les boites contenant la souche $\mathrm{S}_{1}$ sont incubées à $37^{\circ} \mathrm{C}$ et celles contenant les souches $\mathrm{S}_{2}$ et $\mathrm{S}_{3}$ à $30^{\circ} \mathrm{C}$.

Analyse statistique : L'analyse statistique des données collectées a été réalisée avec le logiciel XLSTAT version 7.5.2. Les résultats des activités enzymatiques et de la thermophilie ont été soumis à une analyse de variance (ANOVA) suivi de celle de Student-Newman-Keuls afin de comparer les moyennes de la vitesse de croissance apicale. Les résultats ont été exprimé en moyenne $\pm S D$ et les mesures ont été répétée trois fois $(n=3)$, La différence a été considérée statistiquement significative lorsque la valeur de $p$ est $\leq 0.05$.

gélose de Pomme de Terre Dextrose (PDA) tandis que la souche $S_{3}$ a présenté de petites colonies rondes de 15 millimètres de diamètre (Figure 1). 


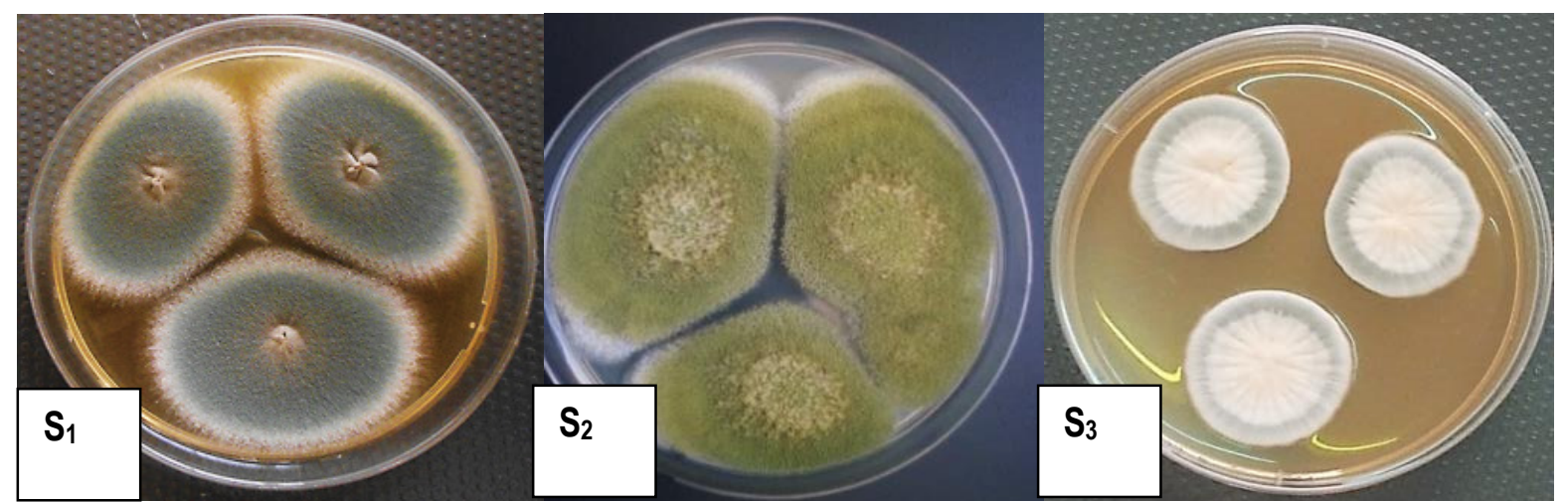

Figure 1 : Aspect macroscopique des trois souches de moisissures sur gélose de Pomme de Terre Dextrose (PDA) incubées à $30^{\circ} \mathrm{C}$ pendant 7 jours (Compaoré et al., 2016b)

Caractérisation microscopique: Au microscope optique les souches $S_{1}$ et $S_{2}$ ont présenté de nombreuses conidies réfringentes avec des conidiophores non

cloisonnés. La souche $S_{3}$ a présenté de rares conidies portées par des conidiophores cloisonnés (Figure 2).

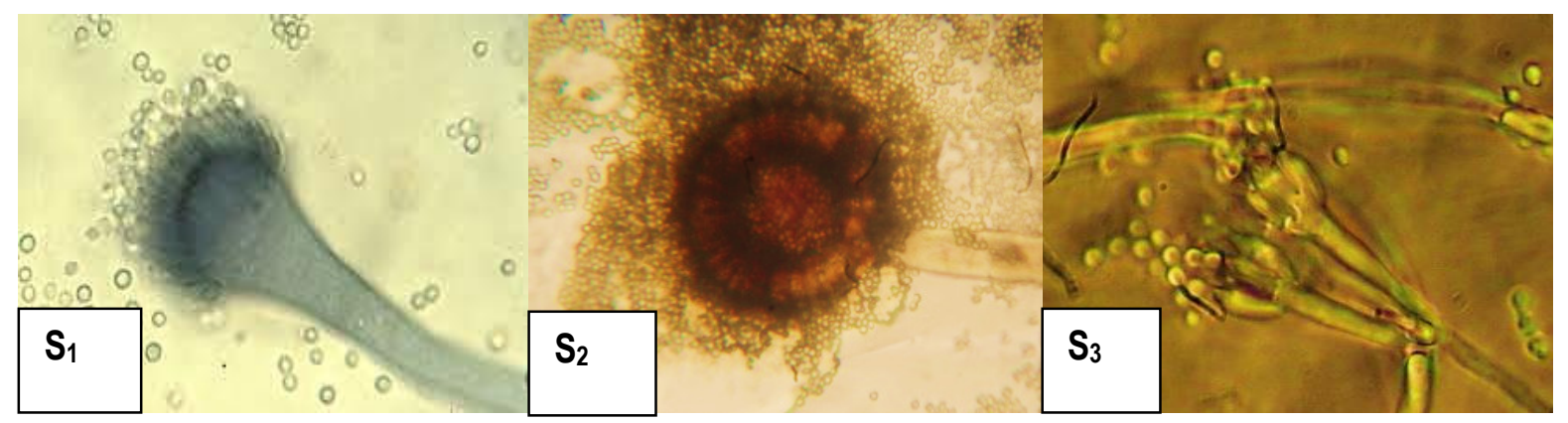

Figure 2 : Aspect microscopique des trois souches de moisissures (x100) (Compaoré et al., 2016b)

Étude de la thermophilie: La figure 3 montre la croissance apicale des différentes souches en fonction de la température d'incubation. La température optimale de croissance est de $37^{\circ} \mathrm{C}$ pour $\mathrm{S}_{1}$ et $30^{\circ} \mathrm{C}$ pour $\mathrm{S}_{2}$ et $\mathrm{S}_{3}$.

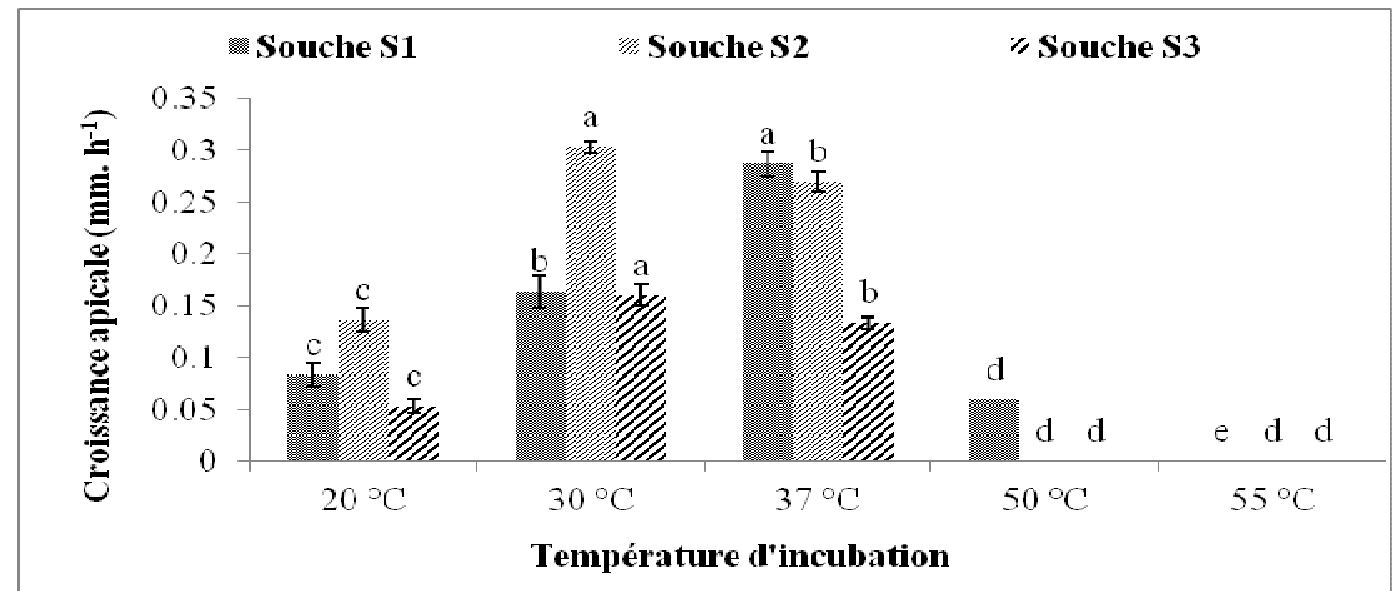

Pour la même souche de moisissure, les valeurs qui ont en commun une même lettre ne sont pas significativement différentes selon le test de Newman-Keuls au seuil de 5\%. Niveau de signification pour les trois souches $S_{1}, S_{2}$ et $S_{3}(p<0,0001)$.

Figure 3 : Croissance apicale $\left(\mathrm{mm} . \mathrm{h}^{-1}\right)$ des trois souches de moisissures 
Aptitude des souches de moisissures à produire des enzymes extracellulaires : Les trois souches ont montré une croissance sur la quasi-totalité des milieux testés avec une croissance maximale sur le milieu à base de glucose. La figure 4 montre la croissance apicale en $\mathrm{mm} \cdot \mathrm{h}^{-1}$ des souches de moisissures sur les différents milieux synthétiques.

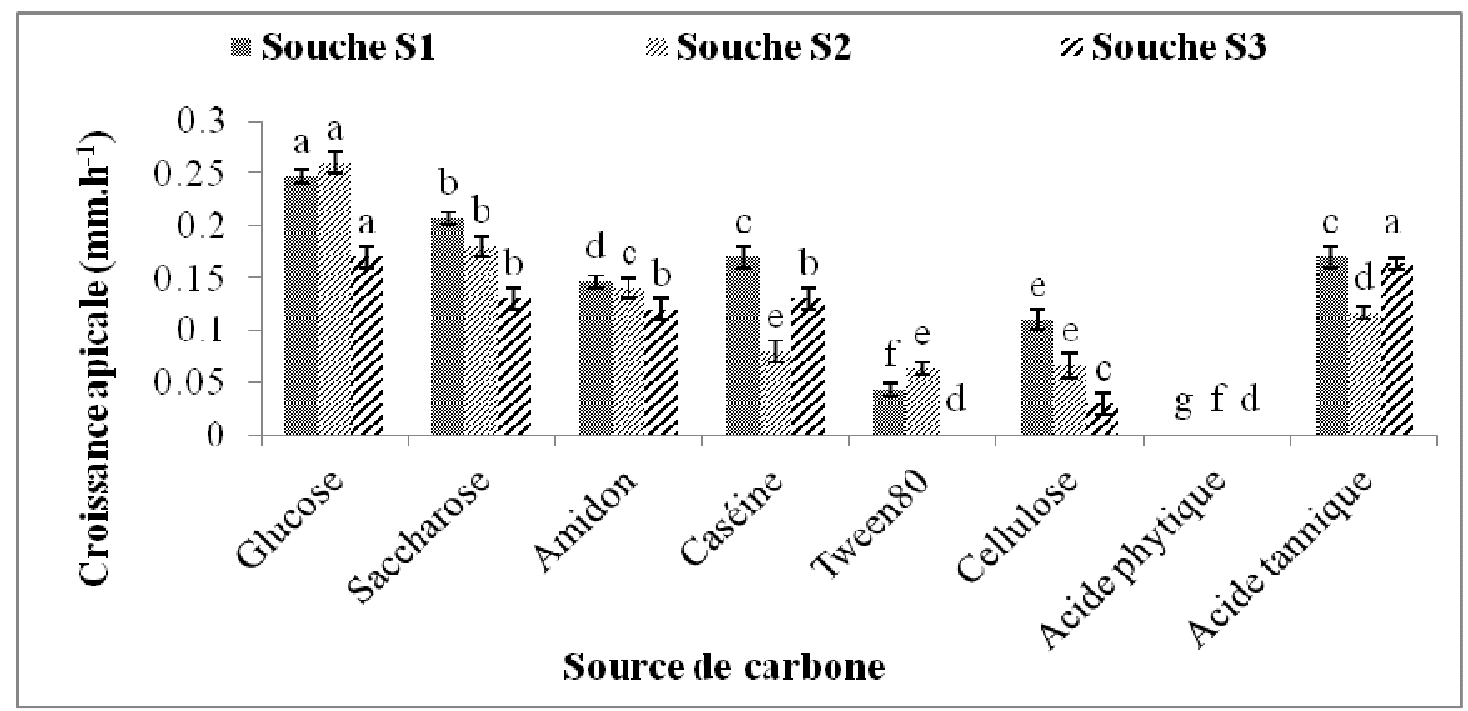

Pour la même souche de moisissure, les valeurs qui ont en commun une même lettre ne sont pas significativement différentes selon le test de Newman-Keuls au seuil de 5\%. Niveau de signification pour les trois souches $S_{1}, S_{2}$ et $S_{3}(p<0,0001)$.

Figure 4 : Croissance apicale $\left(\mathrm{mm}^{\mathrm{h}} \mathrm{h}^{-1}\right)$ des trois souches de moisissures en fonction des différentes sources de carbone, incubées à $37^{\circ} \mathrm{C}$ pour $S_{1}$ et à $30^{\circ} \mathrm{C}$ pour $S_{2}$ et $S_{3}$.

\section{DISCUSSION}

L'identification morphologique des souches $S_{1}$ et $S_{2}$ a été réalisée grâce à la clé de Raper \& Fennell (1965), tandis que la souche $S_{3}$ a été identifiée grâce à la clé de Pitt (1985). Les caractéristiques morphologiques des souches $\mathrm{S}_{1}, \mathrm{~S}_{2}$ et $\mathrm{S}_{3}$ sont respectivement proches de celles d'Aspergillus fumigatus, d'Aspergillus flavus et de Penicillium citrinum (Figures 1 et 2) (Compaoré et al., $2016 b)$. La détermination de la souche $S_{1}$ a été confirmée par la clé de Cooney \& Emerson (1964) pour l'identification des Champignons Filamenteux Thermophiles (CFT). En effet Aspergillus fumigatus est la seule espèce du genre Aspergillus capable de se développer à $50^{\circ} \mathrm{C}$ (Cooney \& Emerson, 1964; Lamrani, 2009). L'étude de la thermophilie montre que la souche $\mathrm{S}_{1}$ isolée du maïs est capable de se développer dans une large gamme de température entre $20^{\circ} \mathrm{C}$ et $50^{\circ} \mathrm{C}$ avec une croissance apicale optimale de $0,28 \mathrm{~mm} \cdot \mathrm{h}^{-1}$ à $37^{\circ} \mathrm{C}$. Cette souche peut être considérée comme thermotolérante car elle présente une croissance à $20^{\circ} \mathrm{C}$. Les souches $S_{2}$ et $S_{3}$ se développent entre $20^{\circ} \mathrm{C}$ et 37 ${ }^{\circ} \mathrm{C}$ avec une croissance apicale optimale respectivement de $0,30 \mathrm{~mm} \cdot \mathrm{h}^{-1}$ et $0.15 \mathrm{~mm} \cdot \mathrm{h}^{-1}$ à $30^{\circ} \mathrm{C}$ (Figure 3). Des résultats similaires avaient été obtenus par Lamrani (2009) sur des souches de moisissures isolées d'une unité de trituration au Maroc. L'étude de la croissance en fonction de la source de carbone montre que les souches sont capables de dégrader une variété de sources de carbone à $37^{\circ} \mathrm{C}$ pour $\mathrm{S}_{1}$ et à $30^{\circ} \mathrm{C}$ pour $\mathrm{S}_{2}$ et $\mathrm{S}_{3}$ (Figure 4). Les trois souches utilisent le glucose, le saccharose, la caséine, l'amidon, la cellulose et l'acide tannique, ce qui suggère qu'elles sont capables de produire des protéases, de la saccharase, des amylases, la cellulase et la tannase. Ces résultats sont en accord avec ceux de Sarah (2011) qui a prouvé que les souches d'Aspergillus flavus, d'Aspergillus fumigatus et de Penicillium Sp étaient capables de se développer sur la cellulose des supports papier. Aspergillus flavus et Aspergillus fumigatus ont dégradé le tween 80 donc peuvent être capables de produire des lipases extracellulaires. Ces résultats corroborent aussi ceux obtenus par Zerroug (2011) qui a remarqué que les champignons endophytes pouvaient produire une panoplie d'enzymes comme les estérase, les protéases, les lipases et l'amylase. En outre Bhat et al. (1998) ont montré que les souches d'Aspergillus flavus et de Penicillium citrinum pouvaient élaborer des tannases. Plusieurs champignons endophytes du genre Aspergillus sont exploités pour leurs capacités à élaborer de l'amylase, des lipases et des 
protéases (Maria et al., 2005). Aucune de ses trois souches ne s'est développée sur le milieu à base d'acide phytique, ce qui suggère qu'elles ne produisent pas des phytases ou bien qu'elles les produisent mais elles sont inactives à $37^{\circ} \mathrm{C}$ pour $S_{1}$ et à $30{ }^{\circ} \mathrm{C}$ pour $S_{2}$ et $S_{3}$. En effet Berka et al. (1998) ont montré que la phytase de Thermomyces lanuginosus avait une activité maximale à $65^{\circ} \mathrm{C}$ tandis que celle d'Aspergillus niger est inactive à cette température. La croissance apicale optimale des trois souches est voisine de $0,2 \mathrm{~mm} \cdot \mathrm{h}^{-1}$, elle a été notée

\section{CONCLUSION}

Dans le cadre du screening de souches de moisissures locales aptes à élaborer des enzymes extracellulaires sur milieu solide, soixante-seize (76) échantillons d'aliments locaux ont été collectés dans des marchés centraux de trois villes du Burkina Faso. Au total Deux cent soixantedouze (272) souches de moisissures ont été isolées sur le milieu PDA et soumises au test indirect de production d'enzymes extracellulaires. Ce screening a révélé l'activité protéolitique, amylolitique, tannasique, lipolitique et cellulosique de trois souches $S_{1}, S_{2}$ et $S_{3}$. En effet, ces enzymes sont très utilisées dans différents domaines. Les tannases sont utilisés en industrie agroalimentaire, les phytases sont utilisés directement comme complément dans les rations alimentaires pour les animaux monogastriques. Les lipases sont utilisées indirectement comme biocatalyseurs dans des applications de Biocatalyse en Chimie Fine. L'étude de la croissance en fonction de la température a montré que la souche $S_{1}$ est thermotolérante tandis que $S_{2}$ et $S_{3}$ sont mésophiles.

\section{REFERENCES BIBLIOGRAPHIQUES}

Awad G, 2005. Caractérisation et étude de l'effet des sources de carbone et d'azote sur la production de nouveaux métabolites secondaires chez Aspergillus ochraceus non producteur de l'ochratoxine A. Thèse de Doctorat, Institut National Polytechnique de Toulouse, Toulouse $222 \mathrm{pp}$.

Berka R, Rey M, Brown KM, Byun T, Klotz AV, 1998. Molecular characterization and expression M.W. of a phytase gene from the thermophylic fungus Thermomyces lanuginosus. Appl. Environ. Microbiol., 64: 4423-4427.

Bhat TK, Bhupinder-Singh O, Sharma P, 1998. Microbial degradation of tannins. A current perspective Biodegradation, 9: 343-357.

Botton $B$, Breton AM, Fèvre $S$, Gauthier $P$, Guy J-P, Larpent P, Reymond J-J, Sanglier Y, Vayssier $P$, Veau, 1999. Moisissures utiles et nuisibles. dans le milieu à base de glucose (Figure 4). En effet plusieurs auteurs ont montré que les sources de carbones rapidement fermentescibles telles que les monosaccharides permettent une bonne croissance du mycélium fongique (Awad, 2005). Cet effet avait été mis en évidence au cours de la production de la pénicilline (Guiraud, 2003). La présence du glucose inhibe la synthèse de l'antibiotique bien que favorisant la croissance du microorganisme et du mycélium (Compaoré et al., 2016b).

L'identification morphologique des souches fongiques grâce aux différentes clés d'identification par l'étude des caractères macroscopiques et microscopiques a révélé que les trois souches $S_{1}, S_{2}$ et $S_{3}$ sont respectivement proches d'Aspergillus fumigatus, d'Aspergillus flavus et de Penicillium citrinum. En effet plusieurs auteurs ont montré que les espèces appartenant aux genres Aspergillus et Penicillium sont capables de produire une panoplie d'enzymes extracellulaires ce qui les confère un fort pouvoir contaminant (Scriban, 1993; Lamrani, 2009). A la lumière des résultats obtenus, on peut conclure que les souches de moisissures isolées d'aliments locaux du Burkina Faso, possèdent d'intéressantes activités enzymatiques. Les résultats obtenus sont encourageants et méritent d'être poursuivis par des essais de production d'enzymes au laboratoire afin d'apprécier la quantité élaborée par unité de temps. L'identification morphologique des souches doit être confirmée par la méthode de la biologie moléculaire.

Importance industrielle. (ed.) Masson Paris, 12$426 \mathrm{pp}$.

Compaoré $H$, Sawadogo-Lingani $H$, Guira F, Waré LY, Samandoulougou S, Savadogo A, Dianou D, Traoré SA, 2016a. Optimization of antimicrobial compound production by Aspergillus fumigatus isolated from maize in Ouagadougou, Burkina Faso. Current Research in Microbiology and Biotechnology, 4 (4): 903-911.

Compaoré $H$, Sawadogo-Lingani $H$, Guira $F$, Samandoulougou S, Savadogo A, Dianou D, Traoré SA, 2016b. Enhancement of antibacterial compounds production by Aspergillus flavus and Penicillium citrinum isolated from locals foods in Bobo Dioulasso and Ouahigouya, Burkina Faso. European Journal of Pharmaceutical and Medical Research, 3 (10): 354-363. 
Cooney DG. \& Emerson R, 1964. Thermophilic fungi (ed) W.H. Freeman and Company. An account of their biology, activities and classification.

Davet $P, 1996$. Vie microbienne du sol et production végétales (ed) INRA. Paris.

Guiraud J-P, 2003. Microbiologie alimentaire, (ed.) Duond: Paris., 7-730 pp.

Hankin L. \& Anagnostakis. SL, 1975. The use of solid media for detection of enzyme production by fungi. Mycologia, 67: 597-607.

Kalyani P, Geetha S, Hemalatha KPJ, 2016. Optimization of cultural conditions for improved production and bioactive metabolites by Aspergillus niger (MTTC-961). European Journal of Pharmaceutical and Medical Research, 3 (10): 255-260.

Kosikowski FV, 1988. Dairy foods research papers. Enzyme behavior utilization in dairy technology. J. Dairy Sci., 71: 557-573.

Lamrani K, 2009. Étude de la biodiversité des moisissures nuisibles et utiles isolées à partir des Maâsra du Maroc. Thèse de Doctorat d'État, Université Mohamed V- Agdal, Rabat 213 pp.

Maria GL, Sridhar KR, Raviraja NS, 2005. Antimicrobial and enzyme activity of mangrove endophytic fungi of southwest coast of India. Journal of Agricultural Technology, 1: 67-80.

Pitt JI, 1985. The genus Penicillium, (ed.) Academic Press, $634 \mathrm{pp}$.

Raper KB. \& Fennell DI, 1965. The Genus Aspergillus, (ed) The Williams and Wilkins Company.

Sarah B, 2011. Identification des moisissures et de leurs métabolites secondaires colonisant des supports papiers : évaluation de la toxicité sur des cellules épithéliales respiratoires in vitro. Thèse de doctorat, Université Paris-Est 187 pp.

Scriban R, 1993. Biotechnologie. Technique et Documentation. 4eme (ed.) Lavoisier. Paris. 30$695 \mathrm{pp}$.

Zerroug A, 2011. Métabolites secondaires bioactifs des champignons endophytes isolés de Retama raetam (Forssk). Mémoire de Magistère, 89 pp. 